\title{
Transverse Momentum Dependent Hadron Multiplicities at COMPASS
}

\author{
Nour Makke \\ Dipartimento di Fisica, Università di Trieste and INFN, \\ Sezione di Trieste, via A. Valerio 2 \\ I-34127 Triste, Italy \\ nour.makke@cern.ch
}

Published 29 February 2016

\begin{abstract}
Unpolarised semi-inclusive deep inelastic scattering is receiving a growing interest as a powerful tool to access poorly known transverse momentum dependent parton distributions and fragmentation functions that play a key role in many processes, in particular in the study of the spin structure of the nucleon. These functions can be investigated through experimental observables. New results on these observables by the COMPASS experiment at CERN will be shown and discussed.
\end{abstract}

Keywords: Nucleon momentum structure; SIDIS; transverse momentum.

PACS numbers: 1 2.38.Aw, 13.60.-r, 13.87.Ce, 14.20.-c, 14.65.-q

\section{Introduction}

The study of the nucleon structure has achieved a high precision level in the determination of the Parton Distribution Functions (PDFs), using quark to hadron Fragmentation Functions (FFs), known nowadays with a reasonable precision [1] in the collinear framework, i.e. integrating over the intrinsic transverse momentum. The situation for Transverse Momentum Dependence (TMD) of these functions turned out to have been behind the schedule over the last few decades and has not been considered with the same level of priority for the unpolarised case, while the study of polarised TMDs is more advanced. Nowadays, all the precise studies of the nucleon spin strongly suggest that the spin fraction carried out by quarks and gluons is relatively small and that the spin fraction carried by orbital angular momentum must be significant, which may imply a significant transverse momentum of quarks. Consequently, a growing interest in the determination of the Transverse Momentum Dependent (TMD) PDFs and FFs is ongoing on both experimental and theoretical levels.

This is an Open Access article published by World Scientific Publishing Company. It is distributed under the terms of the Creative Commons Attribution 3.0 (CC-BY) License. Further distribution of this work is permitted, provided the original work is properly cited. 
The most viable and promising process in terms of its sensitivity to flavordependent motion of intrinsic quarks inside a nucleon and the mechanism underlying their assembling to form uncolored bound states is the Semi-Inclusive Deep Inelastic Scattering (SIDIS). In the framework of perturbative QCD (pQCD) and in the current fragmentation region, the SIDIS cross section factorizes into the product of the short-distance virtual photon-quark hard scattering and the subsequent quark hadronisation. While the hard scattering matrix elements can be computed perturbatively, any information about PDFs and FFs needs to be extracted from experimental data. The TMD-PDFs encode details about the motion of quarks inside a nucleon due to their intrinsic transverse momentum (denoted $k_{\perp}$ ), and the TMD-FFs encode details about producing a hadron with a non-zero transverse component of hadron momentum (denoted $p_{h \perp}$ ) with respect to the fragmenting quark momentum. In addition to the many advantages that SIDIS offers as flavor and charge separation and its accessibility to large hadron energy fraction, it covers a relatively wide energy-scale range, crucially important for $Q^{2}$ evolution of PDFs and FFs in general.

In this paper, a recent measurement of transverse momentum dependent charged hadron multiplicities is presented. It uses a data set collected by the COMPASS collaboration at CERN by scattering a $160 \mathrm{GeV} / \mathrm{c}$ muon beam off a deuteron target.

\section{Data Analysis}

Hadron multiplicity is the most suitable observable allowing to access TMD parton densities and fragmentation functions simultaneously. In the TMD factorisation scheme, the spin-averaged differential cross section for producing final-state hadron with transverse momentum $p_{T}$ and fractional energy $z$ in semi-inclusive deep inelastic scattering, at leading order in $\alpha_{s}$ and $\left(k_{\perp} / Q\right)$, reads [3]:

$$
\begin{gathered}
\frac{d \sigma^{h}}{d x_{B} d Q^{2} d z d p_{T}^{2}}=\frac{2 \pi^{2} \alpha^{2}}{\left(x_{B} s\right)^{2}} \frac{\left[1+(1-y)^{2}\right]}{y^{2}} F_{U U} . \\
F_{U U}=\sum_{q} e_{q}^{2} \int d^{2} k_{\perp} d^{2} p_{h \perp} f_{q / p}\left(x_{B}, k_{\perp}\right) D_{h / q}\left(z, p_{h \perp}\right) \delta^{(2)}\left(p_{T}-z k_{\perp}-p_{h \perp}\right) .
\end{gathered}
$$

where $q$ denotes the quark flavor and $e_{q}$ is the quark charge, $f_{q / p}$ denotes TMDPDFs and $D_{h / q}$ denotes TMD-FFs. The transverse momentum dependence has historically been described assuming a Gaussian parameterisation $\exp \left(-b p_{T}^{2}\right)$, where $b^{-1}$ represents the average transverse momentum squared. In the $\gamma^{*}-p$ c.m. frame, the measured transverse momentum $\left(p_{T}\right)$ is generated by $k_{\perp}$ and $p_{\perp}$ with $\left\langle p_{T}^{2}\right\rangle=$ $\left\langle p_{\perp}^{2}\right\rangle+z^{2}\left\langle k_{\perp}^{2}\right\rangle$. In such case, the TMD-PDFs and TMD-FFs can be parameterrised versus $\left(x_{B}, k_{\perp}\right)$ and $\left(z, p_{\perp}\right)$ respectively as in [4].

Experimentally, a hadron multiplicity is defined (in eq.3) as the average density number of hadrons produced per Deep-Inelastic Scattering (DIS) event. It is defined 
by the number of hadrons produced in a selected data sample normalised to the number of selected DIS events in this sample.

$$
M^{h}\left(x_{B}, Q^{2}, z, p_{T}^{2}\right)=\left(\frac{d^{2} n^{D I S}\left(x_{B}, Q^{2}\right)}{d x_{B} d Q^{2}}\right)^{-1} \cdot \frac{d^{4} n^{h}\left(x_{B}, Q^{2}, z, p_{T}^{2}\right)}{d x_{B} d Q^{2} d z d p_{T}^{2}} .
$$

It can be extracted in bins of different kinematic variables providing a multivariate experimental data appropriate for phenomenological extraction of TMDs. Extracted from data, the hadron multiplicity is subject to many effects: limited kinematic and geometric acceptance effects, smearing effects, electromagnetic radiative effects and should be corrected for all of them. The correction for geometric acceptance and smearing effects is the highest correction and is evaluated using a Monte Carlo (MC) simulation composed of a physics generator, a GEANT based apparatus simulation and finally an algorithm for track reconstruction identical to the one used for experimental data. As the MC simulation contains both the generated and reconstructed properties of all recorded tracks, the acceptance correction factor, $\epsilon^{h}$, is defined by the ratio of the reconstructed hadron yield to the generated one, both recorded from reconstructed DIS sample. It is evaluated in simultaneous bins of $x_{B}$ and $Q^{2}, z$ and $p_{T}^{2}$ as shown in eq. 4 , where $i(j)$ refers to the bin number based on the values of reconstructed (generated) kinematic variables of lepton and hadron MC tracks:

$$
\epsilon^{h}\left(x_{B}, Q^{2}, z, p_{T}^{2}\right)=\frac{n_{r}^{h}\left(i_{x_{B}}, i_{Q^{2}}, i_{z}, i_{p_{T}}\right)}{n_{g}^{h}\left(i_{x_{B}}, i_{Q^{2}}, j_{z}, j_{p_{T}}\right)}
$$

The overall acceptance correction factor is $\leq 0.35$. The radiative effects, which introduce a bias to the kinematics of affected DIS interactions, are corrected for in $x_{B}$ and $y$ only. They are the highest at large $y\left(y>0.7 \sim x_{B}<0.01\right)$. Finally, an additional correction of the contribution of diffractive vector meson production was evaluated. However the results shown in sec. 3 are not corrected for this latter.

A previous measurement [6] of this observable was performed by the COMPASS collaboration using the data set collected in 2004 by scattering a $160(\mathrm{GeV} / \mathrm{c})$ muon beam off an isoscalar ${ }^{6} \mathrm{LiD}$ target, and had a limited kinematic coverage $(0.004<$ $\left.x<0.12, Q^{2}<10(\mathrm{GeV} / \mathrm{c})^{2}, p_{T}^{2}<1.3(\mathrm{GeV} / \mathrm{c})^{2}\right)$ due to the limited angular acceptance of the apparatus. This new measurement uses the data set recorded in 2006 with the same beam energy and target and covers a wider kinematic range $\left(0.004<x<0.7, Q^{2}>1(\mathrm{GeV} / \mathrm{c})^{2}, p_{T}^{2}<3(\mathrm{GeV} / \mathrm{c})^{2}\right)$ and profits from the larger angular acceptance of the apparatus upgraded in 2005. The first results of this analysis were discussed in [7] and the complete set of results is shown and discussed in the following. COMPASS has also measured $p_{T}$-integrated multiplicities for charged hadrons, pions and kaons [8] which have been included in the recent global QCD analysis of FFs in [1]. These results are in excellent agreement with the current measurement, after integration over $p_{T}^{2}$, as expected. Finally the kinematic 


\section{N. Makke}
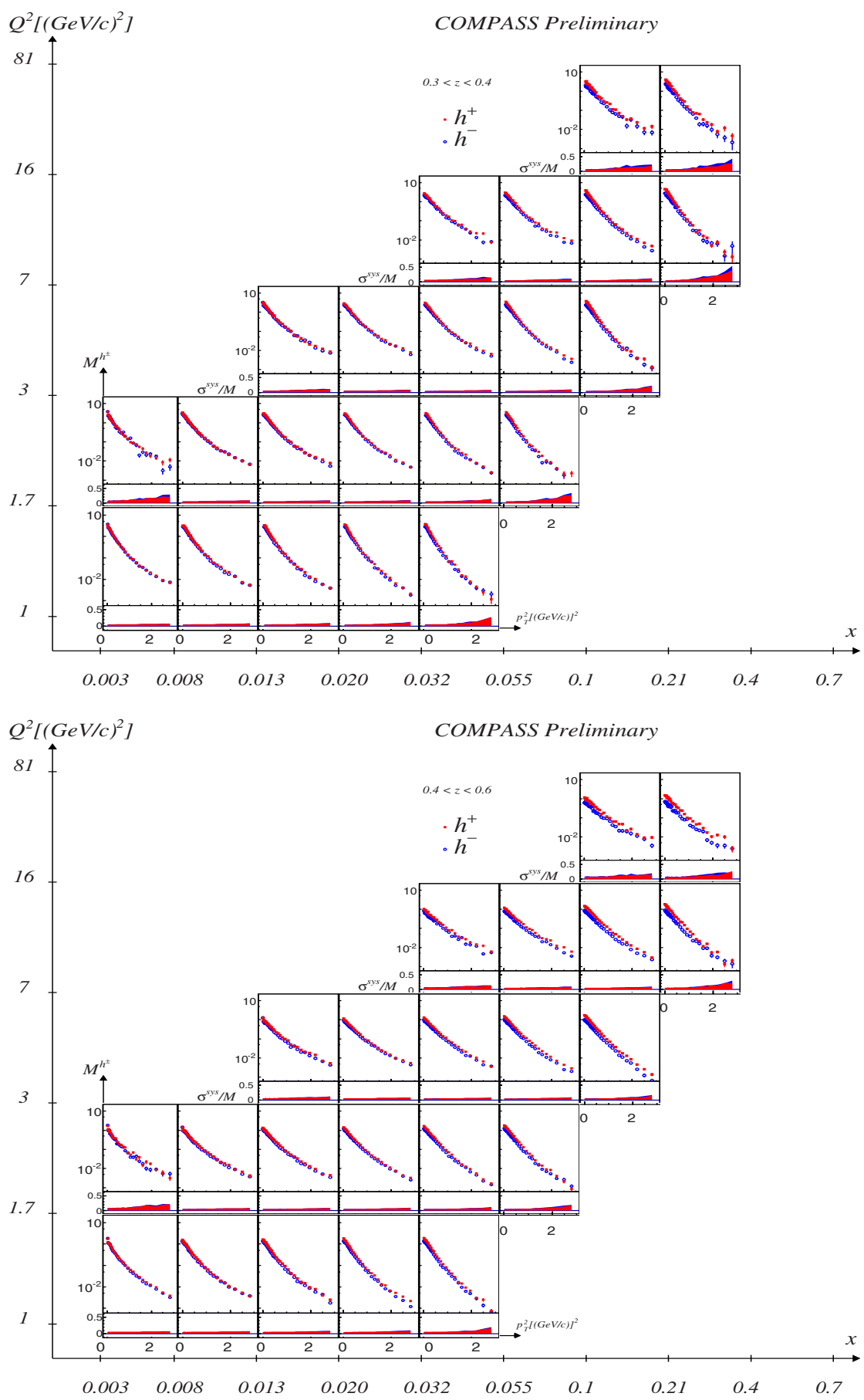

Fig. 1. $h^{ \pm}$multiplicities versus $p_{T}^{2}$ in $\left(x, Q^{2}, z\right)$ bins. $x \equiv x_{B}$. Systematic errors are shown by the bands. Only two $z$ bins are shown here. 

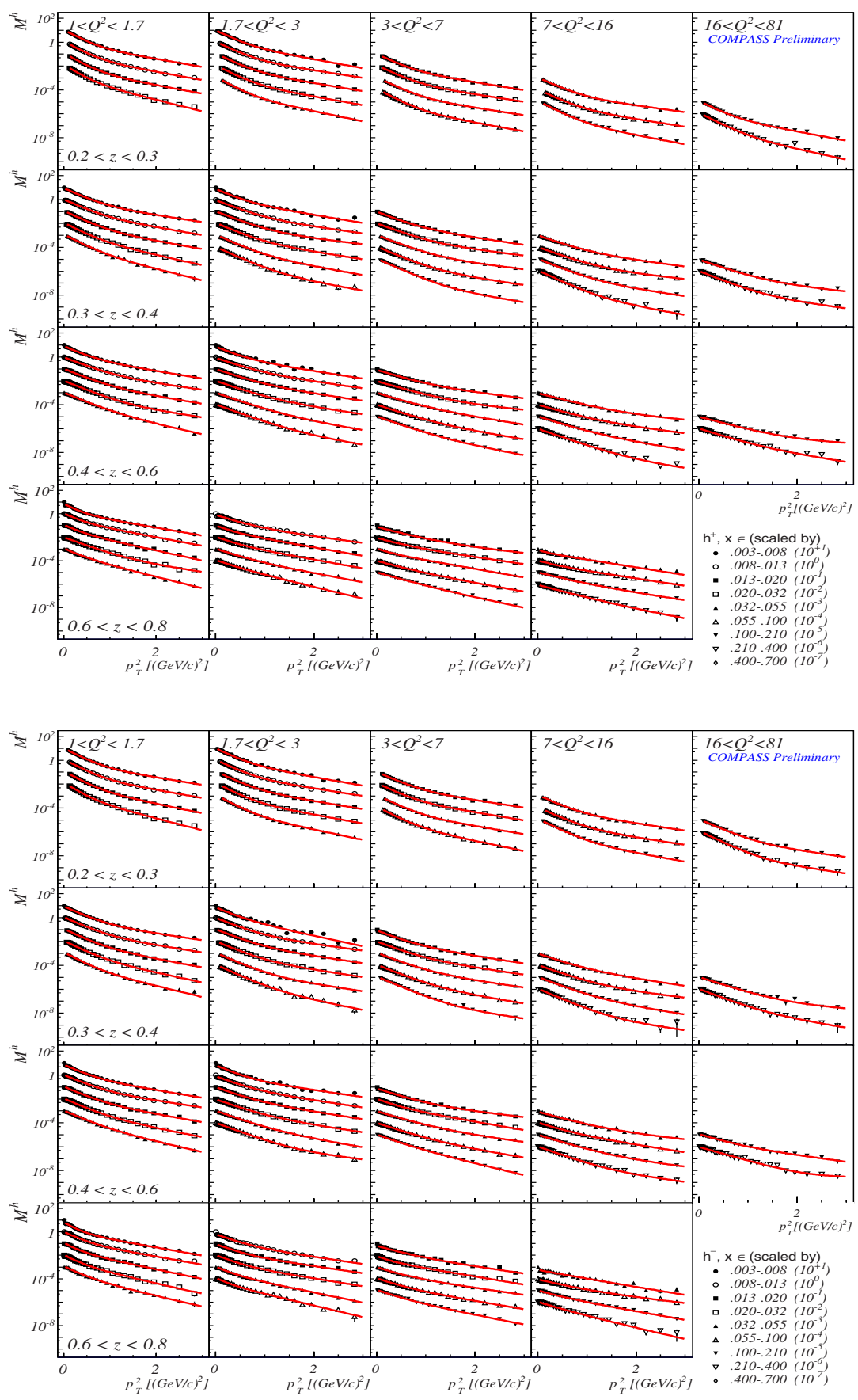

Fig. 2. $h^{+}$(top) and $h^{-}$(bottom) multiplicities versus $\left(x_{B}, Q^{2}, z, p_{T}^{2}\right)$ with 2-exponentials $\left(a e^{-\alpha p_{T}^{2}}+b e^{-\beta p_{T}^{2}}\right)$ fits in the range $p_{T}^{2}<3(\mathrm{GeV} / \mathrm{c})^{2} . x \equiv x_{B}$. Only statistical errors are shown and used in the fit. 
range selected by the current analysis is $Q^{2}>1(\mathrm{GeV} / \mathrm{c})^{2}, W>5(\mathrm{GeV} / \mathrm{c}), y \in$ $[0.1,0.9]$ and $x_{B} \in[0.003,0.7]$.

\section{Results}

The resulting hadron multiplicities are shown in Figs. 1 for both charges as a function of $p_{T}^{2}$ (up to $3(\mathrm{GeV} / \mathrm{c})^{2}$ ) in two $z$ ranges (all results can be found in 10 ). They consist of 4918 experimental data points and represent a key input to phenomenological extraction of TMDs. A difference between positive and negative hadron multiplicities is clearly visible and is more pronounced at large $x$ and large $z$. The difference at large $x$ can be explained by the dominance of valence quarks in positive hadrons while the difference versus $z$ is related to the difference between favored and unfavored hadron FFs.

Nowadays, the understanding of TMDs in unpolarised processes is not yet complete and these results, together with azimuthal asymmetries measured in unpolarised SIDIS $[11,12,13]$, represent a very valuable input to phenomenology extraction of TMDs [4] as well as to test the approximations and hypotheses currently assumed as Gaussian parameterisation. For the results presented here, the Gaussian approximation with constant ( $x$ and/or $z$ independent) mean transverse momentum square is not well suited, especially with the large $p_{T}^{2}$ coverage. A two-exponential function $\left(a \cdot e^{-\alpha p_{T}^{2}}+b \cdot e^{-\beta p_{T}^{2}}\right)$ was instead used and the experimental data were better fitted with this function. The fits are shown in Fig. 2 for positive (top) and negative (bottom) hadrons.

\section{Acknowledgments}

The author undertook this work with the support of the "ICTP TRIL Programme. Trieste, Italy".

\section{References}

1. D. de Florian, R. Sassot, M. Epele, R. J. Hernandez-Pinto and M. Stratmann, arXiv:1410.6027 [hep-ph].

2. P. Abbon et al. [COMPASS Collaboration], Nucl. Instrum. Meth. A 577, 455 (2007).

3. A. Bacchetta, M. Diehl, K. Goeke, A. Metz, P. J. Mulders and M. Schlegel, JHEP 0702, 093 (2007).

4. M. Anselmino et al., JHEP 1404, 005 (2014).

5. A. Airapetian et al. [HERMES Collaboration], Phys. Rev. D 87, 074029 (2013).

6. C. Adolph et al. [COMPASS Collaboration], Eur. Phys. J. C 73, no. 8, 2531 (2013).

7. N. Makke [COMPASS Collaboration], Phys. Part. Nucl. 45, 138 (2014).

8. N. Makke [COMPASS Collaboration], PoS DIS 2013, 202 (2013).

9. N. Makke [COMPASS Collaboration], [arXiv:1411.4244].

10. N. Makke, Talk at SPIN 2014.

11. C. Adolph et al. [COMPASS Collaboration], Nucl. Phys. B 886, 1046 (2014).

12. A. Airapetian et al. [HERMES Collaboration], Phys. Rev. D 87, 012010 (2013).

13. M. Osipenko et al. [CLAS Collaboration], Phys. Rev. D 80, 032004 (2009). 